\title{
The transcription factors KNIRPS and KNIRPS RELATED control cell migration and branch morphogenesis during Drosophila tracheal
}

\section{development}

\author{
Chao-Kung Chen ${ }^{1}$, Ronald P. Kühnlein ${ }^{1}$, Karsten G. Eulenberg ${ }^{1}$, Stéphane Vincent ${ }^{2}$, Markus Affolter ${ }^{2}$ and \\ Reinhard Schuh ${ }^{1, *}$
}

${ }^{1}$ Max-Planck-Institut für biophysikalische Chemie, Abt. Molekulare Entwicklungsbiologie, Am Fassberg, D-37077 Göttingen, Germany

2Biozentrum, Abt. Zellbiologie, Universität Basel, Klingelbergstr.70, CH-4056 Basel, Switzerland

*Author for correspondence

Accepted 1 October; published on WWW 12 November 1998

\section{SUMMARY}

Cell migration during embryonic tracheal system development in Drosophila requires DPP and EGF signaling to generate the archetypal branching pattern. We show that two genes encoding the transcription factors KNIRPS and KNIRPS RELATED possess multiple and redundant functions during tracheal development. knirps/knirps related activity is necessary to mediate DPP signaling which is required for tracheal cell migration and formation of the dorsal and ventral branches. Ectopic knirps or knirps related expression in lateral tracheal cells respecifies their anteroposterior to a dorsoventral migration behavior, similar to that observed in the case of ectopic DPP expression. In dorsal tracheal cells knirps/knirps related activity represses the transcription factor SPALT; this repression is essential for secondary and terminal branch formation. However, in cells of the dorsal trunk, spalt expression is required for normal anteroposterior cell migration and morphogenesis. spalt expression is maintained by the EGF receptor pathway and, hence, some of the opposing activities of the EGF and DPP signaling pathways are mediated by spalt and knirps/knirps related. Furthermore, we provide evidence that the border between cells acquiring dorsal branch and dorsal trunk identity is established by the direct interaction of KNIRPS with a spalt cis-regulatory element.

Key words: knirps, spalt, Drosophila melanogaster, Tracheal system, Cell migration, Morphogenesis

\section{INTRODUCTION}

Directed cell migration is an essential process during the development of multicellular organisms. This process involves an integrated interplay between locally distributed signaling molecules, their receptors and intracellular signal transduction pathways (Hynes and Lander, 1992; Montell, 1994; Lauffenburger and Horwitz, 1996). A model for study of the molecular mechanisms underlying directional cell migration and branch formation is the developing Drosophila tracheal system. It originates from ten metamerically ordered placodes on each side of the embryo. These placodes, which consist of about 90 ectodermal cells each, invaginate in a coordinated manner into the underlying mesoderm and form a stereotyped pattern of tubular structures. Cell migration is the predominant mechanism during branch formation, since tracheal development is a cell-proliferation-independent process. Some of the primary branches grow along the dorsoventral body axis to establish the dorsal, the lateral and the ganglionic branches. An additional set of primary branches extends along the anteroposterior axis to form the dorsal trunk and the visceral branches. The individual tracheal metameres on each side of the embryo eventually connect by fusion of the dorsal trunk and the lateral trunk branches. Unicellular secondary branches are subsequently formed and the two halves of the system become interconnected into a three-dimensional network by anastomosis formation. Finally, additional fusion and terminal branching lead to the highly branched tracheal network, which starts its function, the transport of gases, during the first larval instar stage (for detailed descriptions see Manning and Krasnow, 1993; Samakovlis et al., 1996).

During primary branch outgrowth, three different signaling pathways play a prominent role in directed tracheal cell migration. branchless (bnl), which codes for an FGF-like secreted molecule (BNL; Sutherland et al., 1996), is expressed in cell clusters surrounding the tracheal placodes. Its activity promotes guided tracheal cell migration, a process mediated by the FGF receptor tyrosine kinase BREATHLESS (BTL; Klämbt et al., 1992; Lee et al., 1996). In contrast to BNL signaling, which prefigures tracheal cell migration of all primary tracheal branches, the signaling molecules SPITZ (SPI) and DPP are required for anteroposterior and 
dorsoventral tracheal cell migration, respectively. SPI is ubiquitously expressed and processed into a potent ligand by the transmembrane proteins RHOMBOID (RHO) and STAR (Schweitzer and Shilo, 1997). RHO is tightly regulated and its expression is confined to the central part of the tracheal placode (Wappner et al., 1997). Secreted SPI activates the EGF receptor (EGFR) signaling pathway in the tracheal placodes, which results in anteroposterior tracheal cell migration (Llimargas and Casanova, 1997; Wappner et al., 1997). DPP is expressed dorsally and ventrally of the developing placodes. The dorsaland ventralmost tracheal cells respond to the DPP signal via the receptor serine-threonine kinases THICK VEINS (TKV) and PUNT (PUT), which ultimately lead to cell migration along the dorsoventral body axis (Affolter et al., 1994; Ruberte et al., 1995; Vincent et al., 1997). Hence, the EGF and DPP pathways are likely to specify directional tracheal cell migration by antagonistic effects and they determine localized gene expression patterns as monitored by spalt (sal) and knirps (kni) marker gene expression (Vincent et al., 1997; Wappner et al., 1997). sal encodes a zinc finger-type transcription factor (SAL), which is expressed in the dorsal parts of all tracheal placodes (Kühnlein et al., 1994). During branch outgrowth, SAL expression persists in the cells of the dorsal trunk anterior and dorsal trunk posterior but decreases in the dorsal branch cells. SAL is essential for dorsal trunk cell migration in the anteroposterior direction, which ultimately leads to dorsal trunk formation (Kühnlein and Schuh, 1996).

Here we show that knirps (kni) and knirps related $(k n r l)$, which encode sequence-related zinc finger transcription factors (Rothe et al., 1989), are expressed in overlapping patterns and share redundant functions during tracheal formation. Regionspecific tracheal kni/knrl expression mediates DPP signaling required for cell migration along the dorsoventral body axis. Ectopic kni/knrl expression in lateral tracheal cells reprograms tracheal cell migration from anteroposterior to a dorsoventral direction. Additionally, kni/knrl represses sal function and can thereby prevent dorsal trunk formation. In wild-type embryos, kni/knrl-dependent sal repression occurs in dorsal tracheal cells and is a prerequisite for normal dorsal branch morphogenesis. kni/knrl-dependent sal repression most likely involves a direct interaction of KNI with sal upstream sequences and results in the establishment of a distinct border between cells that gives rise to dorsal trunk and dorsal branches, respectively.

\section{MATERIALS AND METHODS}

\section{DNA analysis}

Methods of DNA manipulation were performed according to standard protocols (Sambrook et al., 1989). To generate UAS-kni, the kni coding region of cDNA-cJ15 (Rothe et al., 1989) was cloned in the P-element vector pUAST (Brand and Perrimon, 1993). The resulting pUAST-kni plasmid was used for P-element mediated transformation of flies to generate UAS-kni effector lines. Electrophoretic mobilityshift assays were carried out as described (Kühnlein et al., 1997) and purified KNI ( $80 \mathrm{ng} / \mu \mathrm{l})$ from bacterial extracts was provided by $\mathrm{R}$. Rivera-Pomar.

\section{Fly stocks}

The following mutant fly lines were used in this study: $k n i^{F C 13}, r h o^{7 M}$, $s p i^{A 14}, f b^{2 W 74}, f l b^{2 C 82}, s a l^{445}, D f(3 L) r i^{X T 1}$ (Lindsley and Zimm, 1992).
$D f(3 L) r i^{X T 1}$ is a deletion which uncovers both $k n i$ and $k n r l$ (Rothe et al., 1989). The kni transgene on the second chromosome, which rescues the kni segmentation phenotype (Rothe et al., 1992) and the kni primary branching phenotype (this work), was used to generate the fly line kni-transgene/CyO;Df(3L)ri ${ }^{X T 1} / \mathrm{TM} 2$ (designated $D f r i{ }^{X T 1}$ kni-transgene). The kni region-specific tracheal enhancer construct drives lacZ expression in the region-specific kni expression domains (Wimmer, 1995). To drive Gal4 ubiquitously in the tracheal system from stage 10 onwards we used btl-Gal4 driver fly lines (Shiga et al., 1996). We applied the following Gal4-dependent UAS effector fly lines: UAS-dpp (Ruberte et al., 1995); UAS-kni (this work); UASknrl (kindly provided by M. Hoch); UAS-NLS-GFP-lacZ for nuclear $\beta$-galactosidase expression (Shiga et al., 1996); UAS-sal (Kühnlein and Schuh, 1996).

\section{Immunostainings}

Whole-mount immunostainings were performed as described (Goldstein and Fryberg, 1994). The secondary antibody was revealed either by the horseradish peroxidase Elite ABC kit (Vector Laboratories) or by staining for alkaline phosphatase activity. To stain tracheal lumen the monoclonal antibody $2 \mathrm{~A} 12$ was used (DSHB, Iowa). To visualize $\beta$-galactosidase a monoclonal antibody (Promega) or a polyclonal antibody (Cappel) was applied. The anti-KNRL rabbit antiserum was provided by M. Hoch and the anti-SAL antibody was described previously (Kühnlein et al., 1994). Immunostained embryos were viewed with a Zeiss Axiophot microscope. Embryos stained with fluorescent antibodies were analyzed by laser scanning microscopy as described (Kühnlein et al., 1996).

\section{In situ hydridizations}

RNA in situ hybridizations to whole-mount embryos were performed as described (Goldstein and Fryberg, 1994). The RNA probes used in our experiments derived from bnl (Sutherland et al., 1996), btl (Klämbt et al., 1992), kni (Rothe et al., 1989), lacZ and sal (Kühnlein et al., 1994).

\section{Reporter gene constructs and generation of transgenic fly lines}

sal upstream DNA fragments (see Fig. 8C) were cloned into the vector pCaSpeRhs43lacZ (Thummel and Pirrotta, 1992). Transgenic fly lines of the recombinant DNA constructs were generated by P-elementmediated germline transformation and for each construct several independent fly lines were analyzed.

\section{RESULTS}

\section{$k n i$ and $k n r l$ are expressed in overlapping patterns during tracheal branch formation}

The kni and knrl genes encode sequence-related transcription factors, which are expressed in overlapping patterns during embryogenesis, including a posterior expression domain during blastoderm stage (Rothe et al., 1989). Whereas kni activity in this domain is required for abdominal segmentation, $k n r l$ lacks segmentation function due to a $23 \mathrm{~kb}$ primary transcript, which is aborted during the short mitotic cycles at blastoderm stage. However, a knrl minigene rescues the segmentation defects of kni mutant embryos (Rothe et al., 1992). Furthermore, knrl can compensate for the lack of kni activity required for normal stomatogastric nervous system (SNS) formation. Thus, KNI and KNRL carry redundant functions during SNS development (González-Gaitán et al., 1994).

kni expression has also been described during tracheal development (Wimmer, 1995). To examine and eventually distinguish tracheal expression of $\mathrm{kni}$ and $\mathrm{knrl}$, we performed 
in situ embryo staining using a kni-specific hybridization probe and KNRL-specific antibodies. The expression of $k n i$ and KNRL appears during stage 10 in the tracheal placodes (Fig. 1A,D). During primary branch formation expression of $k n i$ and KNRL decreases and restricts to the dorsal- and ventralmost cells as well as visceral branch cells (Fig. 1B,E). kni and KNRL expression persists in the cells of dorsal, visceral, lateral trunk and ganglionic branches (Fig. 1C,F,G). Thus, kni and KNRL are expressed in the same spatio-temporal patterns, suggesting that kni and knrl may also share redundant functions during tracheal development.

\section{Tracheal branch outgrowth is affected in embryos lacking $\mathbf{k n i} / \mathbf{k n r l}$ activity}

DPP signaling is required for the directed migration of dorsal and ventral tracheal cells. It activates kni expression and has been proposed to control target gene expression via KNI (Vincent et al., 1997). To elucidate kni function in dorsal and ventral tracheal cells we examined tracheal formation in kni mutant embryos and in embryos mutant for the deficiency $D f(3 L) r i^{X T 1}$, which uncovers both kni and knrl (Rothe et al., 1989).

In contrast to wild-type embryos, which develop ten tracheal metameres (Fig. 2A), kni and $D f(3 L) r i^{X T 1}$ mutant embryos develop only five (Fig. 2B,C). This result reflects the lack of five abdominal segments in both $k n i$ and $D f(3 L) r i^{X T 1}$ mutant embryos (Rothe et al., 1989). However, while the remaining tracheal metameres in kni mutant embryos develop many aspects of a wild-type branching pattern (Fig. 2B), $D f(3 L) r i^{X T 1}$ mutant embryos develop only rudimentary tracheal metameres, which invaginate but lack primary branching and interconnections (Fig. 2C). This suggests a functional back-up by $k n r l$ activity in kni mutant embryos. Furthermore, the lack of primary branching in $D f(3 L) r i^{X T 1}$ mutant embryos suggests that kni/knrl activity participates in early primary branch outgrowth and hence hampers the analysis of a potential $\mathrm{kni} / \mathrm{knrl}$ function during later stages of branch formation.

To overcome both segmentation and primary tracheal branch defects, we rescued $k n i$ and $D f(3 L) r i^{X T 1}$ embryos by a kni transgene that provides both kni segmentation gene function and kni placode expression (Rothe et al., 1992; Fig. 2D). kni mutant embryos bearing the kni transgene develop a normal number of tracheal metameres as well as a wild-typelike tracheal branching (not shown). In $D f(3 L) r i^{X T 1}$ embryos bearing the kni transgene (Dfri ${ }^{X T 1}$ kni-transgene), dorsal trunk formation is similar to wildtype (Fig. 2E), whereas dorsal branch outgrowth is lacking and lateral trunk fusion occurs only partially. In addition, visceral and ganglionic branches fail to contact the gut and the central nervous system, respectively (Fig. 2F,G). Thus, the regionspecific kni/knrl tracheal expression in the dorsal, ventral and visceral branches is required for their formation. The wildtype-like branch outgrowth in the remaining tracheal anlagen of kni mutant embryos suggests that knrl can substitute for kni activity in such embryos.

To unambiguously demonstrate that kni/knrl activity rather than the activity of another gene uncovered by the $D f(3 L) r i^{X T 1}$ is necessary for branch formation, we performed tissue-specific rescue experiments. UAS-kni or UAS-knrl effector constructs were expressed under the control of a tracheal-specific GAL4 driver (btl-Gal4; see Materials and methods) in $D f r i{ }^{X T 1} \mathrm{kni}$ transgene embryos. Such embryos exhibit wild-type-like dorsal, visceral and gangleonic branch outgrowth as well as lateral trunk formation (Fig. 2H-J and not shown), although the ectopic kni or knrl expression causes a reduced diameter and occasional interruption of the dorsal trunk (Fig. 2H,I and not shown; see also below). This indicates that persisting kni or $k n r l$ expression during tracheal development is sufficient to rescue the lack of branch outgrowth of $D f r i{ }^{X T 1}$ kni-transgene embryos. Thus, kni and knrl share redundant functions during tracheal development and the region-specific tracheal $\mathrm{kni} / \mathrm{knrl}$ expression is indeed a prerequisite for wild-type tracheal branch formation.

\section{$\mathrm{kni} / \mathrm{knrl}$ activity is necessary for directed outgrowth of specific tracheal branches}

The embryonic tracheal development exclusively depends on

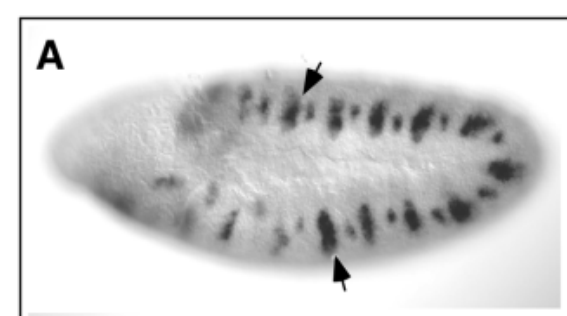

B
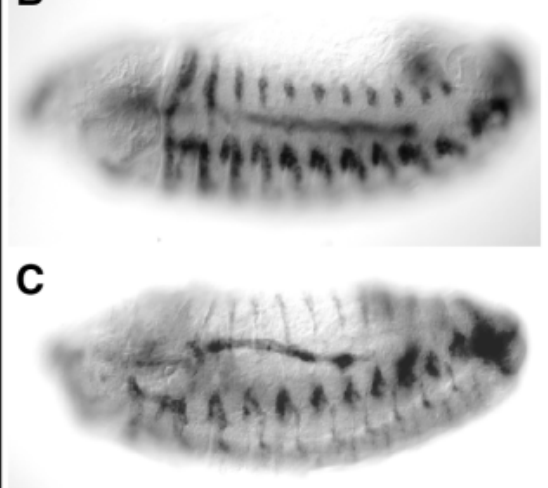

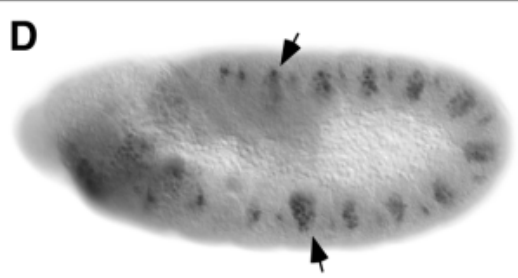

E

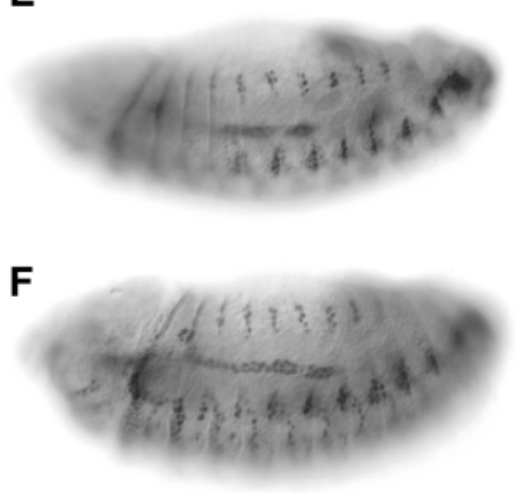

.

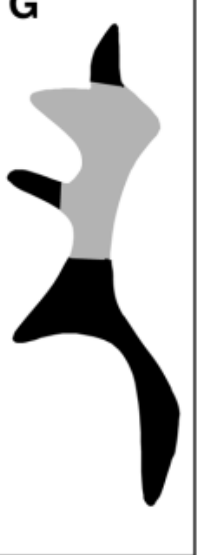

Fig. 1. $k n i$ and KNRL are expressed in overlapping tracheal patterns. Whole-mount in situ hybridization with a kni antisense RNA probe of wild-type embryos at stages 10 (A), 13 (B) and 14 (C). Whole-mount antibody staining with anti-KNRL antibodies of wild-type embryos at stages 10 (D), 13 (E) and 14 (F). The expression of kni (A) and KNRL (D) in the first and tenth tracheal placode is indicated by arrows. (G) Schematic drawing of a tracheal metamere during stage 13; black regions indicate region-specific kni and KNRL expression. Note that KNRL is detected in the cell nuclei. 
Fig. 2. $k n i / k n r l$ activity is necessary for normal tracheal system development. Whole-mount antibody staining for tracheal lumen of stage 15 embryos using the antibody 2A12 (A-C, E-J). (A) Wild-type embryo. (B) $k n i^{F C l 3}$ mutant embryo. The lack of five abdominal segments causes a gap in posterior tracheal structures. (C) $D f(3 L) r i^{X T 1}$ mutant embryo.

(E) $D f(3 L) r i^{X T 1}$ kni-transgene mutant embryo; closeup of metameres 4-6 of a dorsolateral (F) and a ventrolateral region $(\mathrm{G})$. $(\mathrm{H}) D f(3 L) r i^{X T 1}$ kni-transgene mutant embryo bearing the btl-Gal4 driver and the UAS-kni effector; close-up of metameres 4-6 of a dorsolateral (I) and a ventrolateral region (J). Note: The UAS-knrl effector results in equivalent tracheal phenotypes as found for UAS-kni (not shown).

(D) Whole-mount in situ hybridization with a kni antisense RNA probe of a stage $10 D f(3 L) r^{X T 1}$ mutant embryo bearing the kni-transgene. $k n i$ is expressed in the tracheal placodes but no kni expression is detectable in cell groups among the placodes in contrast to wild type (see Fig. 1A). DT, dorsal trunk; LT, lateral trunk; DB, dorsal branch; GB, ganglionic branch; r, rudimentary.

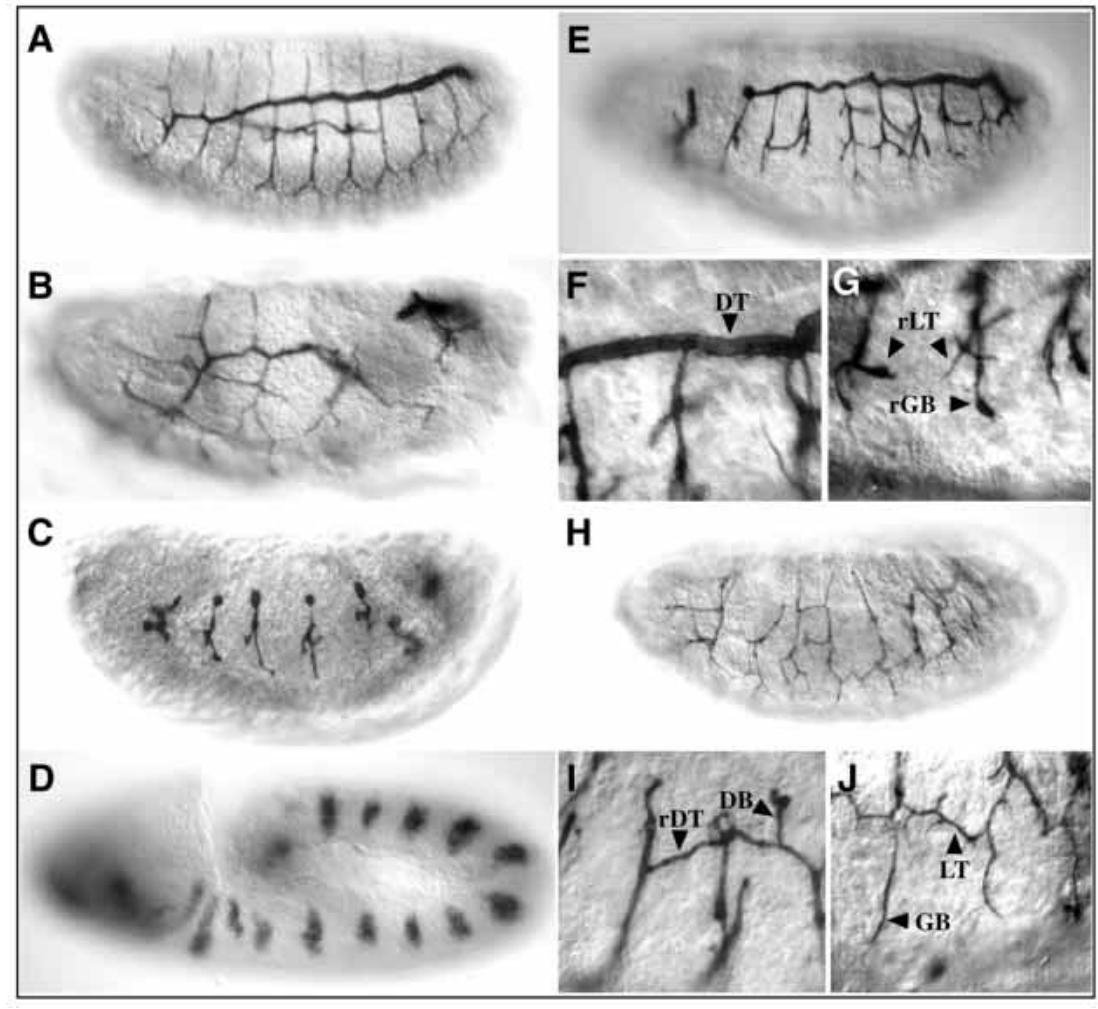

cell migration and cell extension processes (Manning and Krasnow, 1993). Therefore, the lack of specific tracheal structures caused by mutations is either due to cell death or to stalled cell outgrowth. To further characterize the role of

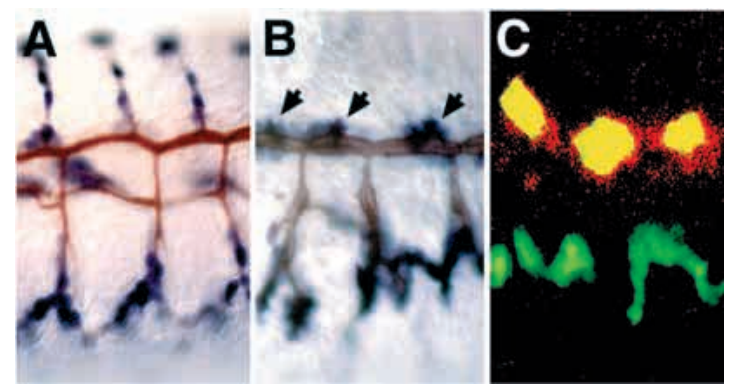

Fig. 3. kni/knrl activity is necessary for branch outgrowth but not for cell viability. Whole-mount antibody double staining with antibody $2 \mathrm{~A} 12$ (brown) and anti- $\beta$-galactosidase antibodies (blue) of wild-type (A) and of $D f r i{ }^{X T 1}$ kni-transgene (B) stage 15 embryos bearing the kni region-specific tracheal enhancer $l a c Z$ construct. The kni regionspecific tracheal enhancer drives $\beta$-galactosidase expression that is indistinguishable from wild-type kni tracheal expression (compare A with Fig. 1C; Wimmer, 1995). (C) Whole-mount antibody double

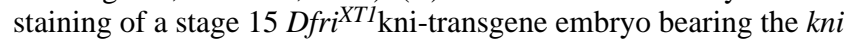
region-specific tracheal lac $Z$ construct using anti-SAL antibodies (red; CY3-coupled secondary antibodies) and anti- $\beta$-galactosidase antibodies (green; fluorescein-coupled secondary antibodies). Superimposition of CY3 and fluorescein pattern (yellow) reveals coexpression of SAL and $\beta$-galactosidase. $\beta$-galactosidase expressing cells of wild-type dorsal branches (A) become integrated in clusters into the dorsal trunk of embryos lacking the region-specific $\mathrm{kni} / \mathrm{knrl}$ expression (arrows in B); these cells express SAL, which serves as a dorsal trunk marker (yellow in $\mathrm{C}$ ). $\mathrm{kni} / \mathrm{knrl}$ during tracheal outgrowth we monitored the fate of tracheal cells marked by reporter gene expression driven by the region-specific kni enhancer (Wimmer, 1995) in both wild-type and $D f r i^{X T l}$ kni-transgene embryos. Reporter gene expression is observed in a kni-like pattern in wild-type tracheae (Fig. 3A). In $D f r^{X T 1}$ kni-transgene embryos the reporter gene product is expressed in the stalled ventral and visceral branches and in cell clusters associated with the dorsal trunk (Fig. 3B). These cell clusters are arranged in a segmentally repeated pattern at dorsal positions of the dorsal trunk where dorsal branches form in wild-type embryos. Thus, the lack of tracheal branch formation in $D f r i^{X T 1}$ kni-transgene embryos is caused by the failure of proper cell migration and not by tracheal cell death.

The close association of $\beta$-galactosidase expressing cells with the dorsal trunk suggests that these cells may participate in dorsal trunk formation in the absence of kni/knrl activity. To confirm this, we examined reporter gene expression in combination with SAL expression, which serves as a marker for dorsal trunk cells (Kühnlein and Schuh, 1996). Interestingly, the dorsal trunk cells coexpress SAL and $\beta$ galactosidase (Fig. 3C), showing that dorsal branch progenitor cells are integrated into the dorsal trunk and express SAL in response to the lack of kni/knrl activity. Thus, the kni/knrl activity is necessary for the directed outgrowth of dorsal, ventral and visceral tracheal cells and it determines dorsal branch cell fate.

\section{$\mathrm{kni} / \mathrm{knrl}$ acts independently of FGF and EGF signaling key components}

FGF signaling controls tracheal branching by guiding tracheal cell migration (Lee et al., 1996; Sutherland et al., 1996). The key component in this process is the chemoattractant BNL, which directs tracheal branches towards their targets 
(Sutherland et al., 1996). The BNL signals are received by the FGF receptor BTL (Klämbt et al., 1992).

We examined whether stalled branch outgrowth in $D f r i{ }^{X T 1}$ kni-transgene embryos is caused by altered $b n l$ and/or $b t l$ expression. In such embryos, bnl expression is the same as in wild-type embryos, including the dorsalmost $b n l$ patches which, however, fail to direct dorsal branch outgrowth in $D f r i{ }^{X T 1}$ kni-transgene embryos (Fig. 4A,B). Furthermore, $D f r i{ }^{X T 1}$ kni-transgene embryos show normal btl expression in their rudimentary tracheal system (Fig. 4C) and region-specific $k n i$ expression is not affected in btl mutant embryos (not shown). Thus, the region-specific kni/knrl activity does not interfere with FGF signaling, although the results do not exclude the possibility that $\mathrm{kni} / \mathrm{knrl}$ may affect downstream components of the FGF pathway.

The EGF pathway is activated by the generation of activated SPITZ (SPI) in the tracheal placode and is necessary for dorsal trunk and visceral branch formation (Wappner et al., 1997). As this visceral branch phenotype is similar to the one of $D f r i{ }^{X T 1}$ kni-transgene embryos, we monitored whether kni expression is still occurring in spi mutant embryos. As shown in Fig. 4D kni expression is normal in spi mutant embryos. Taken together, these results indicate that region-specific kni/knrl expression is not controlled by FGF or EGF signaling and that $\mathrm{kni} / \mathrm{knrl}$ activity does not affect key components of these pathways.

\section{$k n i / k n r l$ activity is necessary but not sufficient to mediate DPP-dependent tracheal patterning}

DPP signaling is required for dorsal and ventral tracheal branch formation and for kni expression (Vincent et al., 1997). The tracheal mutant phenotypes of embryos lacking the DPP

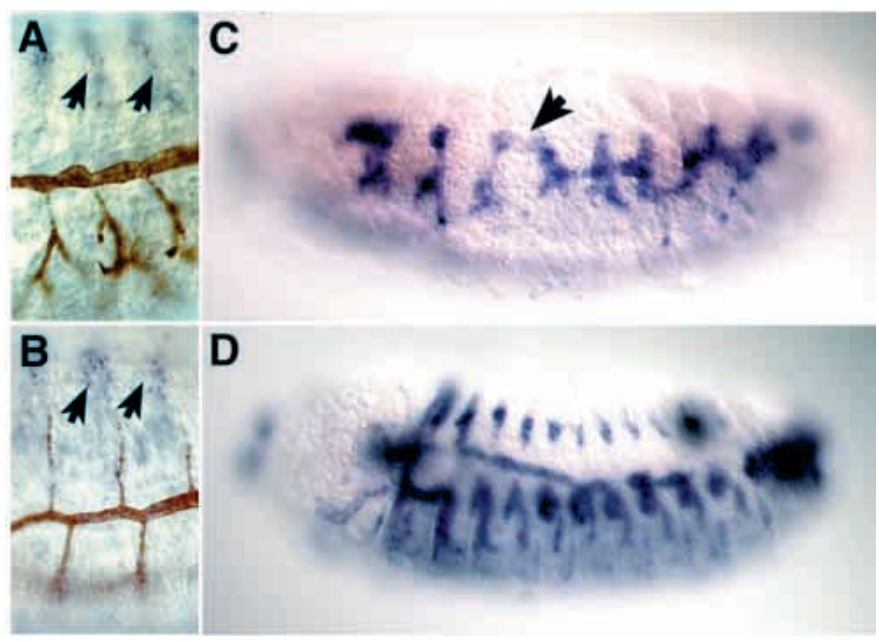

Fig. 4. kni/knrl acts independently of FGF and EGF signaling components. Whole-mount staining with antibody 2A12 (brown) and in situ hybridization with a $b n l$ antisense RNA probe (blue) of $D \mathrm{fri}^{X T 1}$ kni-transgene (A) and wild-type (B) stage 14 embryos. (C) In situ hybridization with a $b t l$ antisense RNA probe (blue) of a

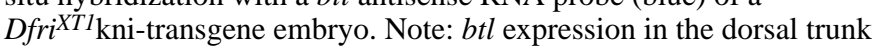
decreases (arrow), as shown for wild type (Ohshiro and Saigo, 1997). (D) In situ hybridization with a kni antisense probe (blue) of a spi mutant embryo. Embryos that lack the region-specific kni/knrl activity lack dorsal branch outgrowth, despite normal bnl (arrows in $\mathrm{A}$ and $\mathrm{B}$ ) and $b t l$ expression (arrow in C). receptors TKV and PUT are reminiscent of the kni tracheal phenotype, suggesting that $k n i$ is necessary to mediate functional aspects of DPP signaling (this work; Vincent et al., 1997). To link kni activity and DPP signaling more directly, we expressed $k n i$ in $t k v$ mutant background by using the trachealspecific btl-Gal4 driver and the UAS-kni effector. Since $t k v$ mutant embryos lack the dorsalmost patches of $b n l$ expression that are necessary for dorsal branch outgrowth (Vincent et al., 1997), we focused our analysis on ventral branch formation in the presence of kni expression. These embryos develop a rudimentary ventral tracheal system that is indistinguishable from the branching of $t k v$ mutants (not shown). Thus, the activation of kni expression by DPP is necessary but not sufficient for ventral branch formation. This result also suggests that DPP signaling controls additional genes different from kni/knrl that are necessary for branch outgrowth (see Discussion).

Ectopic DPP expression in all tracheal cells leads to dorsoventral cell migration, which causes the lack of dorsal trunk and visceral branches that are normally formed by anteroposterior migration (Llimargas and Casanova, 1997; Vincent et al., 1997; Wappner et al., 1997). It also leads to ectopic kni expression in all tracheal cells (Vincent et al., 1997). Our finding that ectopic kni expression also interferes with dorsal trunk formation (Fig. 2H,I) suggests a role of kni activity in mediating ectopic DPP signaling. Thus, we examined the tracheal phenotypes generated by either ectopic $d p p$ or ectopic kni expression. Ubiquitous tracheal $d p p$ expression by btl-Gal4 driver and UAS-dpp effector causes the lack of anterioposterior branch formation and the migration of supernumerary cells towards dorsal (Vincent et al., 1997; compare Fig. 5A,B with 5C,D). Ubiquitous tracheal UAS-kni expression in response to one copy of btl-Gal4 leads to a reduced dorsal trunk and an increased number of cells migrating dorsally (compare Fig. 5A,B with E,F), whereas UAS-kni driven by two copies of btl-Gal4 results in the absence of the dorsal trunk and the migration of supernumary cells towards dorsal positions (compare Fig. 5C,D with G,H). Thus, kni activity leads to a dorsal tracheal cell migration, as observed for DPP.

In summary, our results indicate that the role of DPP in directing tracheal cells to adopt a dorsoventral migration behaviour is mediated by kni/knrl activity, but kni/knrl is not sufficient to mediate DPP-dependent branch formation.

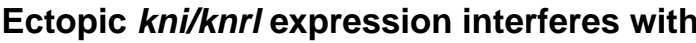 anteroposterior cell migration by repressing sal}

To gain insight into the mechanisms of kni/knrl-dependent reprogramming of tracheal cells from anteroposterior to dorsoventral migration, we first examined the activities of genes known to be required for dorsal trunk formation. In embryos mutant for genes of the EGFR pathway the dorsal trunk is strongly affected and the visceral branches are reduced, suggesting a role of this pathway in specifying anteroposterior tracheal cell migration (Wappner et al., 1997). In sal mutant embryos dorsal trunk progenitor cells grow out in an erratic manner resulting in a tracheal phenotype reminiscent of EGFR signaling mutant phenotypes, with the difference that sal mutant embryos exhibit normal visceral branch formation (Kühnlein and Schuh, 1996). The observation that sal expression is reduced in the rudimentary tracheal system of rho 
mutant embryos (Wappner et al., 1997) suggests that sal expression may be controlled by EGFR signaling.

To investigate this possibility we monitored sal expression at different stages of tracheal development in embryos that lack components of EGFR signaling. In embryos mutant for rho, spi or faint little ball $(f l b)$, sal expression is not affected during early processes of primary branch formation (stage 11; Fig. $6 \mathrm{~A}, \mathrm{~B}$; not shown). However, during stage 12 sal tracheal expression is strongly reduced in embryos lacking EGFR signaling (compare Fig. 6C with D; not shown) and is not detectable after stage 13 in metameres, which fail to form a dorsal trunk (Wappner et al., 1997; not shown). These results suggest that the EGFR pathway is not necessary for the initiation but for the maintenance of tracheal sal expression.

Ectopic kni expression in dorsal trunk progenitor cells causes a phenotype that is reminiscent of the tracheal phenotypes caused by the lack of EGFR signaling or by the lack of sal, respectively. Hence we tested whether ectopic kni activity interferes with the EGFR pathway or the activation of its transcriptional target sal by examining both the activated state of the EGFR pathway (Wappner et al., 1997) and the SAL expression pattern. The doublephosphorylated form of mitogenactivated protein kinase (dp-ERK) is detectable in similar amounts in wildtype embryos and in embryos that express ectopic kni in response to the btl-Gal4 driver (not shown). Thus, kni does not interfere with the activation of the EGFR pathway in tracheal cells. In contrast, SAL expression is repressed by ectopic kni expression (Fig. 6G-J). UAS-kni expression driven by one copy of btl-Gal4 reduces dorsal trunk SAL expression and dorsal trunk diameter (compare Fig. 6E,F with G,H), whereas UASkni expression driven by two copies of btl-Gal4 causes the lack of SAL expression and dorsal trunk formation (Fig. 6I,J). Thus, kni activity results in repression of sal in dorsal trunk cells and consequently in a lack of dorsal trunk formation. Furthermore, these results also indicate that the lack of anteroposterior dorsal trunk cell migration due to ectopic DPP is mediated by the transcriptional activation of $\mathrm{kni} / \mathrm{knrl}$, which in turn represses SAL.

\section{Dorsal branch development requires SAL repression by kni/knrl activity}

The observation that ectopic kni/knrl activity in the tracheal system leads to the repression of SAL prompted us to ask whether such an interaction is also necessary for normal tracheal development. In wild-type embryos SAL is expressed in the dorsal parts of all tracheal metameres. However, prior to dorsal trunk formation, SAL fades away in the dorsal branch cells and persists in the dorsal trunk cells only (Kühnlein and Schuh, 1996). Double staining experiments show that the decreasing SAL expression correlates with the activation of $\beta$-galactosidase expression driven by the region-specific tracheal kni enhancer in dorsal branch cells (Fig. 7A,B). This suggests that kni/knrl is involved in the repression of sal in dorsal branch cells. Furthermore, the lack of kni expression in dorsal branch progenitor cells of $D f r i{ }^{X T 1}$ kni-transgene embryos results in persistent SAL expression in these cells and their integration into the dorsal trunk (see above). While these observations correlate well with the proposal that kni/knrl activity represses sal in wild-type dorsal branch cells, they do not provide evidence that repression of sal is necessary for normal dorsal branch development. Therefore, we examined dorsal branch

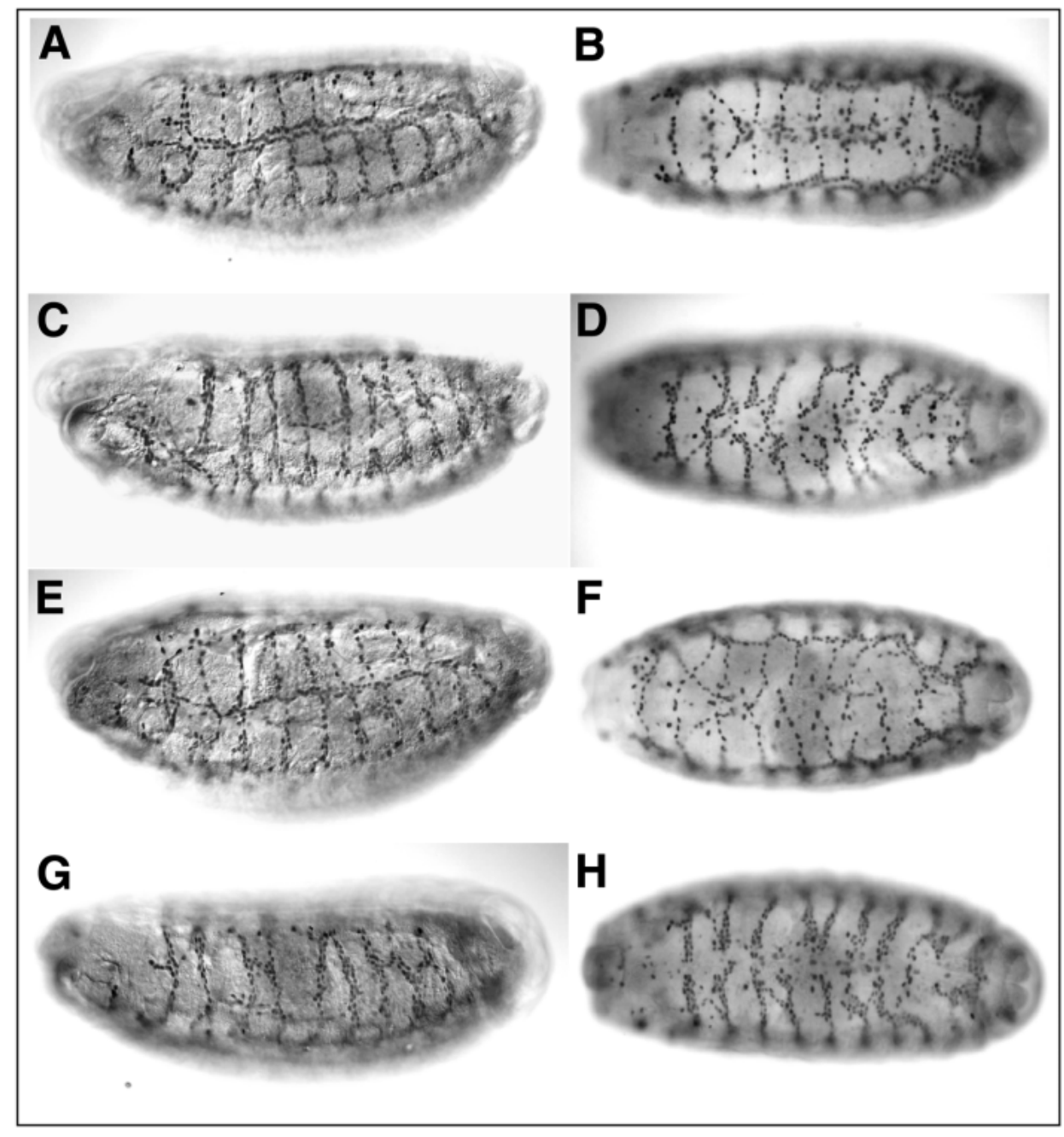

Fig. 5. kni/knrl activity leads to dorsoventral tracheal cell migration. Whole-mount antibody staining with anti- $\beta$-galactosidase antibodies of stage 16 embryos bearing the btl-Gal 4 driver $(A, B)$, the btl-Gal4 driver and the UAS-dpp effector (C,D), the btl-Gal4 driver and the UAS-kni effector (E,F), two copies of the btl-Gal4 driver and the UAS-kni effector $(\mathrm{G}, \mathrm{H})$ and the UASlacZ effector construct, which drives nuclear-localized $\beta$-galactosidase. Embryos are shown in a lateral $(\mathrm{A}, \mathrm{C}, \mathrm{E}, \mathrm{G})$ and dorsal $(\mathrm{B}, \mathrm{D}, \mathrm{F}, \mathrm{H})$ view. Note: UAS-knrl results in equivalent tracheal cell migration as found for UAS-kni (not shown). 
morphogenesis upon continuously providing sal activity via UAS-sal/btl-Gal4. We found that persistent SAL expression in dorsal branch progenitor cells had no effect on their primary outgrowth (compare Fig. 7C with G). However, subsequent development of dorsal branches is affected, i.e. they fail to grow out to the dorsal midline and lack the typical U-turn structure of wild-type dorsal branches (compare Fig. 7D,E with H,I). Furthermore, dorsal anastomosis formation is blocked (compare Fig. 7F with J). This indicates that the repression of sal in dorsal branch progenitor cells is necessary for their normal elongation and for anastomosis formation and it demonstrates that the repression by $k n i / k n r l$ is required for normal dorsal branch development. It is important to note that ectopic SAL in dorsal branch cells does not affect $\mathrm{kni} / \mathrm{knrl}$ expression, indicating that SAL interferes with molecular processes independent and/or downstream of kni/knrl activity under these conditions.

\section{KNI binds in vitro to sal regulatory DNA sequences that direct tracheal expression}

The expression patterns of sal and kni as well as their genetic interaction suggest that sal tracheal expression is directly repressed by KNI in dorsal branch cells. To identify cisregulatory sequences of sal that respond to KNI, we monitored $\beta$-galactosidase reporter gene expression driven by the sal tracheal enhancer (salTSE1000; Fig. 8A,E; Kühnlein and Schuh, 1996; Kühnlein et al., 1997). In embryos that express KNI ubiquitously in the tracheal system, $\beta$-galactosidase expression of salTSE1000 is abolished (not shown) as found for wild-type sal expression. This indicates that the salTSE1000 contains binding sites for KNI-mediated repression. To examine whether KNI directly interacts with the salTSE1000, we performed electrophoretic mobility-shift assays using KNI protein and different DNA-subfragments of the $1 \mathrm{~kb}$ salTSE1000 enhancer region (Fig. 8B). In addition, we delimited the sal tracheal cis-control region by generating transgenic embryos expressing reporter genes under the control of various subfragments of salTSE1000 (Fig. 8C). From five subfragments analyzed, only a 230 bp DNA fragment (salTSE230) binds to KNI (Fig. 8B,D; not shown) and conducts a sal-like $\beta$-galactosidase tracheal expression pattern (Fig. 8C,F). Furthermore, salTSE230-dependent reporter gene expression is repressed by ectopic KNI or DPP (Fig. 8G; not shown). These results indicate that the activation of sal expression in tracheal cells and its KNI-dependent repression in dorsal branch cells are mediated by a 230-bp cis-regulatory element containing sites for a direct interaction with KNI.

\section{DISCUSSION}

\section{$k n i$ and $k n r l$ share redundant functions during tracheal development}

The transcription factors KNI and KNRL are expressed in overlapping patterns during tracheal development. Tissuespecific rescue experiments of kni and knrl deficient embryos by kni- or knrl-expressing transgenes show that the genes share redundant tracheal functions, as has been described for SNS development (González-Gaitán et al., 1994). kni/knrl activity is involved in distinct cellular processes of tracheal cell differentiation. During placode formation, $\mathrm{kni} / \mathrm{knrl}$ is expressed in all tracheal cells. Embryos lacking kni/knrl expression develop rudimentary tracheal branches, a phenotype reminiscent of branchless (Sutherland et al., 1996), breathless (Klämbt et al., 1992) or tracheae defective (Eulenberg and Schuh, 1997) mutants. This suggests that the early tracheal $\mathrm{kni} / \mathrm{knrl}$ activity is required for tracheal development prior to primary branch formation.

In contrast to early $\mathrm{kni} / \mathrm{knrl}$ expression in all tracheal cells, $\mathrm{kni} / \mathrm{knrl}$ is expressed in a region-specific manner during primary branch formation in a subset of the major branches. The absence of kni/knrl expression in visceral branch primordial cells results in rudimentary visceral branches, as has been observed in mutants lacking components of the EGF pathway (Llimargas and Casanova, 1997; Wappner et al., 1997). However, this aspect of kni/knrl expression is not dependent on EGF signaling (unpublished results and Wappner et al., 1997), suggesting that EGF signaling and $\mathrm{kni} / \mathrm{knrl}$ activity function in parallel. Embryos that lack the dorsal and ventral $\mathrm{kni} / \mathrm{knrl}$ expression lack branches which grow out dorsally (dorsal branch), whereas branches that extend ventrally (ganglionic branch, lateral trunk) are rudimentary. This phenotype is reminiscent of mutants affecting DPP signaling and supports previous observations suggesting that dorsal and ventral kni expression are activated by DPP (Vincent et al., 1997). Moreover, the finding that ectopic $\mathrm{kni} / \mathrm{knrl}$ expression is able to reprogram tracheal cell migration along the dorsoventral body axis and causes cells to migrate in a dorsal direction, is similar to the altered migration in response to ectopic DPP (Vincent et al., 1997). Thus, the proposed role of DPP to regionalize the tracheal placode by selecting groups of cells that adopt specific migration behaviors appears to be mediated by kni/knrl activity. However, rescue experiments by artificial $k n i$ expression in homozygous $t k v$ mutant embryos does not overcome the lack of DPP signaling in the tracheal system. Therefore, kni/knrl activity is necessary but not sufficient to mediate DPP signaling in the tracheal system and indicates that additional DPP-dependent genes are required for dorsal and/or ventral branch outgrowth. For ventral branch formation, unplugged (unp) represents such a candidate gene. unp is necessary for ganglionic branch morphogenesis, but its DPP dependence has not yet been investigated (Chiang et al., 1995).

\section{Repression of sal by $k n i / k n r l$ controls dorsal branch morphogenesis}

$\mathrm{kni} / \mathrm{knrl}$ activity is necessary for the initiation of dorsal branch formation, i.e. the migration of dorsal branch cells towards the dorsal side of the embryo (see above). In addition, $k n i$ plays a role in subsequent dorsal branch development by repression of sal activity in these cells. sal repression is essential for normal dorsal branch formation, as shown by ectopic SAL expression experiments. Whereas the migration of dorsal branch cells is not affected, proper dorsal branch morphogenesis does not occur in the presence of SAL. In wild-type embryos the six (approx.) dorsally migrating cells are of cuboidal shape and arranged side-byside at embryonic stage 12 . Up to stage 15 , the branches continue to extend to the dorsal side and the cells elongate and intercalate to an end-to-end organization. The two leading dorsal branch cells, DB1 and DB2, develop long 

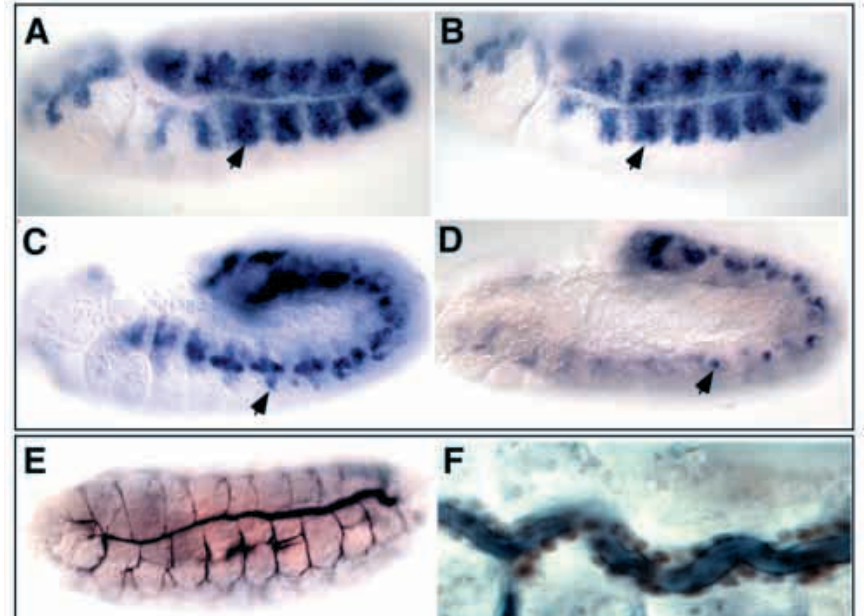

G
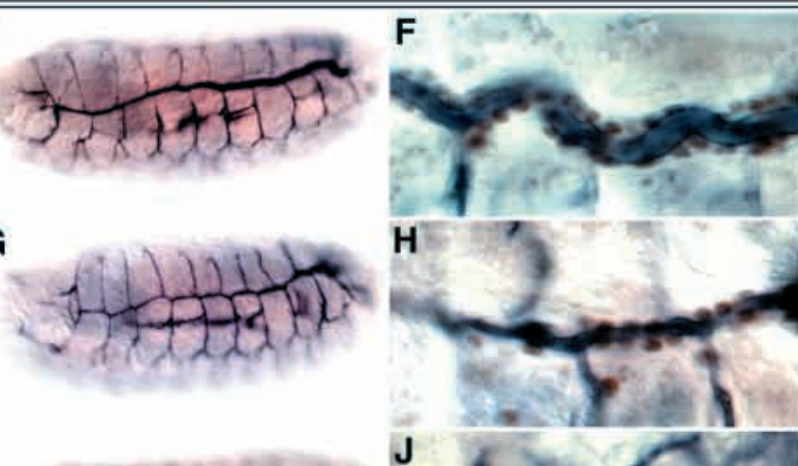

I

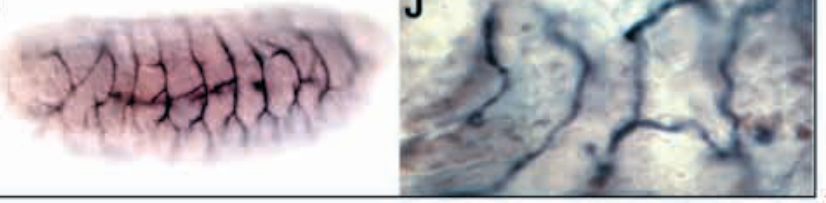

Fig. 6. Ectopic $k n i / k n r l$ expression in tracheal cells interferes with dorsal trunk development and represses SAL. Whole-mount in situ hybridization with a sal antisense RNA probe of wild-type $(\mathrm{A}, \mathrm{C})$ and rho ${ }^{7 M}$ mutant embryos (B,D) at stage 11 (A,B) and stage 12 (C,D). Arrows in A and B point to sal expression in the first tracheal metamere of the wild-type (A) and the $r h o^{7 M}$ (B) mutant embryo. Arrows in $\mathrm{C}$ and $\mathrm{D}$ point to sal oenocyte expression, which is present in the wild-type (C) and the $r h o^{7 M}$ (D) mutant embryo; but note that the $r h o^{7 M}$ embryo lacks tracheal sal expression in the corresponding metamere. Whole-mount antibody staining with the antibody $2 \mathrm{~A} 12$ (E,G,I) or antibody double staining with the antibody 2A12 (blue) and anti-SAL antibodies (brown; F,H,J) of wild-type embryos (E,F), of embryos bearing the btl-Gal4 driver and the UAS-kni effector $(\mathrm{G}, \mathrm{H})$ and of embryos bearing two copies of the btl-Gal4 driver and the UAS-kni effector (I,J). Note: UAS-knrl results in similar repression of SAL as found for UAS-kni (not shown).

cytoplasmic extensions, termed secondary branches. The extension of the DB1 cell approaches the dorsal trunk, whereas the DB2 branch grows out toward the dorsal midline and fuses with its contralateral partner, thus forming the dorsal anastomosis (Samakovlis et al., 1996). sal activity interferes with several developmental steps of dorsal branch formation. In the presence of SAL cell elongation and intercalation is delayed. Furthermore, dorsal anastomosis formation and outgrowth of the DB1 cell back to the dorsal trunk fails and normal terminal branching of DB1 cells during stage 16 was not detectable in response to SAL. Thus, ectopic SAL interferes with dorsal branch extension and it prevents secondary and terminal branch formation. Since tracheal cell intercalation, secondary and terminal branch formation are not found during dorsal trunk morphogenesis (Samakovlis et al., 1996) it is likely that a function of SAL

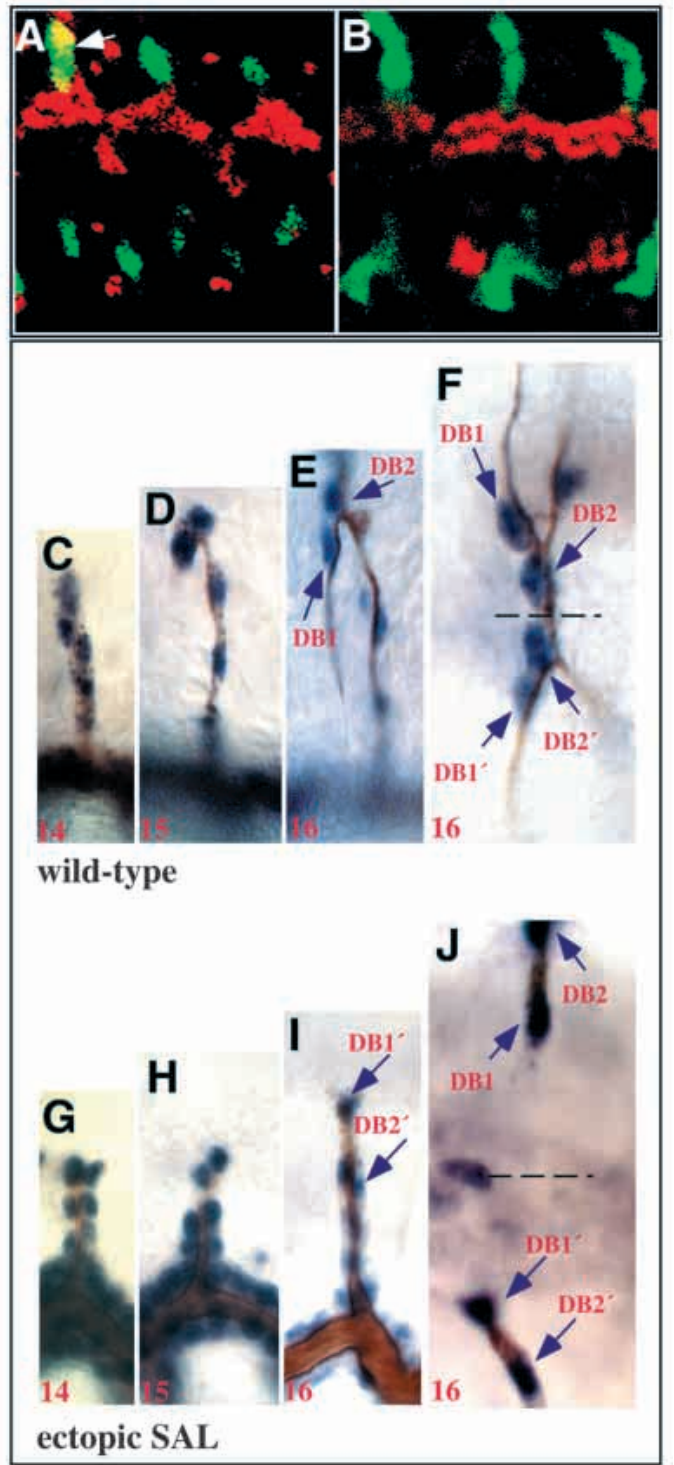

Fig. 7. Ectopic SAL expression causes rudimentary dorsal branch formation and lack of dorsal anastomoses. Whole-mount double staining using anti-SAL antibodies (red; CY3-coupled secondary antibodies) and anti- $\beta$-galactosidase antibodies (green; fluoresceincoupled secondary antibodies) of a stage 12 (A) and a stage 13 (B) embryo, both bearing the kni region-specific tracheal lac Z construct. Superimposition of CY3 and fluorescein pattern (yellow) reveals coexpression of SAL and $\beta$-galactosidase in dorsal branch cells (arrow in A). Whole-mount antibody double staining with antibody $2 \mathrm{~A} 12$ (brown) and anti- $\beta$-galactosidase antibodies (blue) of stage-14 $(\mathrm{C}, \mathrm{G})$, stage $15(\mathrm{D}, \mathrm{H})$ and stage $16(\mathrm{E}, \mathrm{F}, \mathrm{I}, \mathrm{J})$ embryos bearing the btlGal4 driver and the UAS-lacZ effector constructs (C-F) and bearing in addition the UAS-sal effector construct (G-J). (C-E and G-I) Dorsolateral view of single dorsal branches of tracheal metamere 5; $(\mathrm{F}, \mathrm{J})$ dorsal view visualizing a pair of dorsal branches of tracheal metamere 5; developmental stages are numbered. DB, dorsal branch cell. Arrows in E,F and I,J point to the nuclei of terminal dorsal branch (DB1 and DB2) cells. The dashed lines in F and J mark the dorsal midline.

is to prevent these processes during normal dorsal trunk development. This assumption is supported by mutant analysis and SAL overexpression studies, which indicate a 


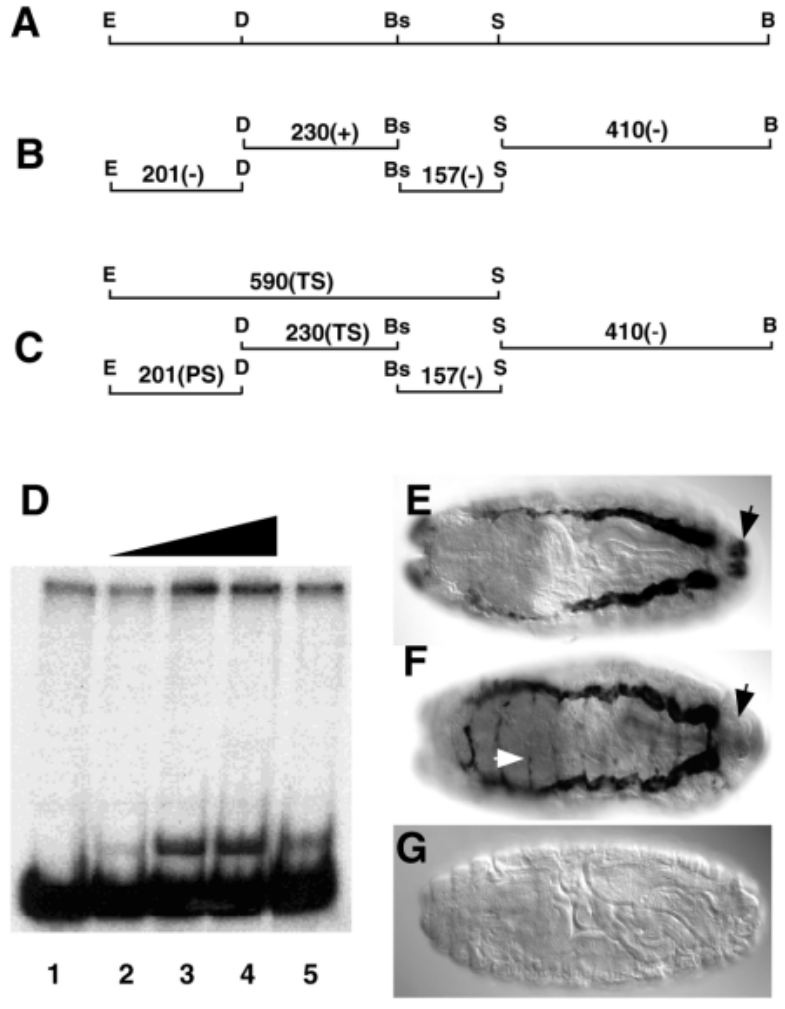

Fig. 8. 230-bp sal cis-regulatory DNA sequences mediate sal tracheal system expression and bind KNI in vitro. (A) Restriction map of the 1 $\mathrm{kb}$ sal tracheal system enhancer (salTSE1000; for expression pattern see E); B, BamHI; Bs, BsaAI; D, DraI; E, EcoR1; S, SpeI. (B) DNA fragments that were used for gel mobility-shift experiments using bacterially expressed and purified KNI are indicated. The fragments E/D201, Bs/S157 and S/B410 show no retardation band (not shown), which is indicated by $(-)$. The fragment $\mathrm{D} / \mathrm{Bs} 230$ shows a retardation band (see D), which is indicated by (+). (C) DNA subfragments that were used for lacZ enhancer assays in transgenic flies. The lack of lacZ expression during embryogenesis driven by the DNA elements S/B410 and $\mathrm{Bs} / \mathrm{S} 157$ is indicated by (-). lacZ expression driven by the element E/D201 in some cells of the developing posterior spiracles (not shown) is indicated by (PS). sal-like lacZ tracheal expression driven by the elements E/S590 and D/Bs230 (see F) is indicated by (TS). (D) Autoradiograph of gel mobility-shift experiments using ${ }^{32} \mathrm{P}$-labeled D/Bs230 DNA and poly(dI-dC) as unspecific competitor DNA lacking KNI (lane 1), with increasing amounts (triangle) of purified KNI (1 $\mu$, lane $2 ; 3 \mu \mathrm{l}$, lane $3 ; 5 \mu \mathrm{l}$, lane 4 ) and $5 \mu$ l purified KNI together with 80 molar excess of unlabeled D/Bs230 DNA as specific competitor (lane 5). (E-G) Whole-mount in situ hybridization with a lacZ antisense RNA probe of stage 16 embryos bearing salTSE1000 (E), bearing lacZ reporter gene driven by D/Bs230 (salTSE230; F) and bearing salTSE230, the btl-Gal4 driver and the UAS-kni effector constructs (G). Note: the staining in $\mathrm{F}$ is developed 3 times longer than in $\mathrm{E}$ to obtain similar strong dorsal trunk expression. Under these staining conditions faint dorsal branch staining of salTSE230 reporter gene expression becomes visible (white arrow in F). Ectopic tracheal kni expression causes repression of salTSE1000 (not shown) and salTSE230 reporter gene expression (G). Reporter gene expression in the posterior spiracles anlagen driven by salTSE1000 (black arrow in E) is strongly reduced in embryos driven by salTSE230 (black arrow in F).

role of sal in the repression of molecular processes that lead to secondary and terminal branch formation (C.-K. Chen and R. Schuh, unpublished). Taken together, these findings establish that repression of sal in dorsal branch cells is required for normal dorsal branch morphogenesis.

Several lines of evidence indicate that $\mathrm{kni} / \mathrm{knrl}$ activity causes the repression of sal. (1) sal expression is detectable during the placode stage in the dorsal half of the tracheal metamers. After kni expression is turned on in dorsal branch primordial cells sal expression gradually decreases during dorsal branch formation. This indicates a strict and inverse spatial and temporal correlation between sal and kni/knrl expression. (2) Dorsal branch primordial cells lacking kni/knrl activity continue sal expression and these cells become integrated into the dorsal trunk. (3) Gain-of-function experiments indicate that ectopic kni/knrl expression represses sal in a concentration-dependent manner in dorsal trunk cells. (4) KNI binds in vitro to sal cis-regulatory sequences that mediate tracheal expression. These findings indicate that kni/knrl-dependent sal repression in dorsal branch cells involves the direct binding of the transcription factors KNI/KNRL.

\section{EGFR signaling maintains sal expression}

sal expression in dorsal tracheal cells is necessary for the migration of dorsal trunk progenitor cells along the anteroposterior axis and for dorsal trunk morphogenesis (Kühnlein and Schuh, 1996). The sal mutant phenotype in the dorsal trunk is similar to the phenotype observed in embryos lacking components of the EGFR pathway (Kühnlein and Schuh, 1996; Llimargas and Casanova, 1997; Wappner et al., 1997). Thus, EGFR and sal may act in the same genetic circuitry. This inference is confirmed by the finding that EGFR signaling is necessary to maintain sal expression in dorsal trunk cells prior to fusion and subsequent morphogenesis, whereas EGFR signaling is not required for the initiation of sal expression. The mutant EGFR pathway causes a lack of tracheal branches to migrate in anteroposterior direction and thus affects the morphogenesis of the dorsal trunk and the visceral branches (Llimargas and Casanova, 1997; Wappner et al., 1997). In sal mutant embryos the dorsal trunk is absent, whereas visceral branches develop normally (Kühnlein and Schuh, 1996). Therefore, sal may function in a patterning system that has been proposed to represent the basis for the distinction between anteroposterior migrating dorsal trunk and visceral branch cells within the domain controlled by EGFR signaling (Wappner et al., 1997).

\section{The DPP and EGF pathways interact via their target genes kni and sal}

It has been proposed that the DPP and EGF signaling generates three different cell fates in the developing placode that confer the capacity to migrate in distinct directions (Wappner et al., 1997). We have shown that kni/knrl activity is necessary to mediate DPP signaling for dorsal and ventral cell migration. In addition, repression of the EGFR signaling target sal by kni/knrl establishes a border between the dorsally and anteroposteriorly migrating dorsal branch and dorsal trunk cells, respectively. However, the repression of sal is not necessary for normal dorsoventral tracheal cell migration but rather for morphogenetic processes that occur independently of cell migration. Thus, tracheal cells that express sal and kni/knrl still adopt a dorsoventral migration behavior. Ectopic expression of $\mathrm{kni} / \mathrm{knrl}$ in dorsal trunk cells has two 
consequences. Firstly, it represses SAL, which results in the lack of anteroposterior migration of dorsal trunk cells. Secondly, ectopic kni/knrl leads primordial dorsal trunk cells to adopt a dorsoventral migration behavior. Thus, the observation that ectopic DPP causes altered tracheal cell migration and lack of dorsal trunk formation (Llimargas and Casanova, 1997; Vincent et al., 1997; Wappner et al., 1997) is consistent with the proposal that these processes are mediated in part via kni/knrl. However, we noted that in contrast to ectopic DPP, which inhibits visceral branch formation, ectopic kni/knrl tracheal expression does not affect anterior outgrowth of visceral branches. This observation is not unexpected since $\mathrm{kni} / \mathrm{knrl}$ is expressed in visceral branch cells and is necessary for normal visceral branch morphogenesis. Thus, kni/knrl acts within the genetic circuitry of visceral branch cell fate determination in a different way from during dorsal branch development and it does not involve mediation of dorsoventral cell migration. The results also show that kni/knrl may be part of a patterning system for visceral branch development within the EGFR signaling domain, whereas sal activity is necessary for dorsal trunk development.

We are grateful to S. Hayashi for btl-Gal4, M. Hoch for UAS-knrl, M. Krasnow for the $b n l$ cDNA, M. Rothe and M. Hoch for the antiKNRL antiserum, R. Rivera-Pomar for purified KNI and B. Shilo for the $b t l$ cDNA. We are indebted to G. Dowe and H. Taubert for expert technical assistance. Special thanks go to H. Jäckle and D. Wainwright for critical reading of the manuscript. We thank $H$. Jäckle for providing a stimulating environment. This work was supported by the Max-Planck-Society (MPIbpc Abt. 170), the SFB 271 (R. P. K. and K. G. E.), the Graduiertenkolleg 'Molekulare Genetik der Entwicklung' (C.-K. C.) and the Swiss National Science Foundation and the Kanton Basel-Stadt (M. A.).

\section{REFERENCES}

Affolter, M., Nellen, D., Nussbaumer, U. and Basler, K. (1994). Multiple requirements for the receptor serine/threonine kinase thick veins reveal novel functions of TGF $\beta$ homologs during Drosophila embryogenesis. Development 120, 3105-3117.

Brand, A. H. and Perrimon, N. (1993). Targeted gene expression as a means of altering cell fates and generating dominant phenotypes. Development 118, 401-415.

Chiang, C., Young, K. E. and Beachy, P. A. (1995). Control of Drosophila tracheal branching by the novel homeodomain gene unplugged, a regulatory target for genes of the bithorax complex. Development 121, 3901-3912.

Eulenberg, K. G. and Schuh, R. (1997). The tracheae defective gene encodes a bZIP protein that controls tracheal cell movement during Drosophila embryogenesis. EMBO J. 16, 7156-7165.

Goldstein, L. S. B. and Fryberg, E. A. (1994). Drosophila melanogster: Practical uses in cell and molecular biology. Meth. Cell Biol. 44, 446-488, 576-598.

González-Gaitán, M., Rothe, M. Wimmer, E. A., Taubert, H. and Jäckle, H. (1994). Redundant functions of the genes knirps and knirps-related for establishment of anterior Drosophila head structures. Proc. Nat. Acad. Sci. USA 91, 8567-8571.

Hynes, R. O. and Lander, A. D. (1992). Contact and adhesive specificities in the associations, migrations, and targeting of cells and axons. Cell 68, 303322.

Klämbt, C., Glazer, L. and Shilo, B. Z. (1992). breathless, a Drosophila FGF receptor homolog, is essential for migration of tracheal and specific midline glial cells. Genes Dev. 6, 1668-1678.

Kühnlein, R.P., Frommer, G., Friedrich, M., Gonzalez-Gaitan, M., Weber, A., Wagner-Bernholz, J. F., Gehring, W. J., Jäckle, H. and Schuh, R. (1994). spalt encodes an evolutionarily conserved zinc finger protein of novel structure which provides homeotic gene function in the head and tail region of the Drosophila embryo. EMBO J. 13, 168-179.

Kühnlein, R.P., Brönner, G., Taubert, H. and Schuh, R. (1997). Regulation of Drosophila spalt gene expression. Mech. Dev. 66, 107-118.

Kühnlein, R. P. and Schuh, R. (1996). Dual function of the region specific homeotic gene spalt during Drosophila tracheal system development. Development 122, 2215-2223.

Lauffenburger, D. A. and Horwitz, A. F. (1996). Cell migration: A physically integrated molecular process. Cell 84, 359-369.

Lee, T., Hacohen, N., Krasnow, M. A. and Montel, D. J. (1996). Regulated Breathless receptor tyrosine kinase activity required to pattern cell migration and branching in the Drosophila tracheal system. Genes Dev. 10, 2912-2921.

Lindsley, D. L. and Zimm, G. G. (1992). The Genome of Drosophila melanogaster. Academic Press, Inc., San Diego.

Llimargas, M. and Casanova, J. (1997). ventral veinless, a POU domain transcription factor, regulates different transduction pathways required for tracheal branching in Drosophila. Development 124, 3273-3281.

Manning, G. and Krasnow, M. A. (1993). Development of the Drosophila tracheal system. In The Development of Drosophila melanogaster (ed. M. Bate and A. Martinez Arias), pp. 609-685. New York: Cold Spring Harbor Laboratory Press.

Montell, D. J. (1994). Moving right along: regulation of cell migration during Drosophila development. Trends Genet. 10, 59-62.

Ohshiro, T. and Saigo, K. (1997). Transcriptional regulation of breathless FGF receptor gene by binding of TRACHEALESS/dARNT heterodimers to three central midline elements in the Drosophila developing trachea. Development 124, 3975-3986.

Rothe, M., Nauber, U. and Jäckle, H. (1989). Three hormone receptor-like Drosophila genes encode an identical DNA-binding finger. EMBO J. 8, 3087-3094.

Rothe, M., Pehl, M. Taubert, H. and Jäckle, H. (1992). Loss of gene function through rapid mitotic cycles in the Drosophila embryo. Nature 359, 156-159.

Ruberte, E., Marty, T. Nellen, D. Affolter, M. and Basler, K. (1995). An absolute requirement for both the type II and type I receptors, punt and thick veins, for dpp signaling in vivo. Cell 80, 889-897.

Samakovlis, C., Hacohen, N., Manning, G., Sutherland, D. C., Guillemin K. and Krasnow, M. A. (1996). Development of the Drosophila tracheal system occurs by a series of morphologically distinct but genetically coupled branching events. Development 122, 1395-1407.

Sambrook, J., Fritsch, E. F. and Maniatis, T. (1989). Molecular Cloning: A Laboratory Manual. Cold Spring Harbor, NY.

Schweitzer, R. and Shilo, B.-Z. (1997). A thousand and one roles for the Drosophila EGF receptor. Trends Genet. 13, 191-196.

Shiga, Y., Tanaka-Matakatsu, M. and Hayashi, S. (1996). A nuclear GFP/ßgalactosidase fusion protein as a marker for morphogenesis in living Drosophila. Dev. Growth Differ. 38, 99-106.

Sutherland, D., Samakovlis, C. and Krasnow, M. A. (1996). branchless encodes a Drosophila FGF homolog that controls tracheal cell migration and the pattern of branching. Cell 87, 1091-1101.

Thummel, C. S. and Pirrotta, V. (1992). New pCaSpeR P-element-vectors. Dros. Inf. Serv. 71, 150.

Vincent, S., Ruberte, E., Grieder, N. C., Chen, C.-K., Haerry, T., Schuh R. and Affolter, M. (1997). DPP controls tracheal cell migration along the dorsoventral body axis of the Drosophila embryo. Development 124, 27412750.

Wappner, P., Gabay, L. and Shilo, B.-Z. (1997). Interaction between the EGF receptor and DPP pathways establish distinct cell fates in the tracheal placodes. Development 124, 4707-4716.

Wimmer, E. A. (1995). Untersuchungen zur Struktur, Funktion und Regulation von buttonhead, einem Kopf-Gap-Gen von Drosophila melanogaster. Thesis, University of Munich, Germany. 